\title{
Interactive comment on "Determining the hierarchical order by which the variables of sampling season, dust outbreaks occurrence, and sampling location, can shape the airborne bacterial communities in the Mediterranean basin" by Riccardo Rosselli et al.
}

\section{Anonymous Referee \#3}

Received and published: 7 February 2021

General Remarks: The manuscript describes a study on the parameters affecting airborne microbial community composition, e.g., season, dust intrusion, geographic proximity to the dust source. These are important questions in the study of aerobiology, especially in the Mediterranean basin that is prone to increasing frequency of Saharan dust intrusions. The study presents a surprising result, according to which the time of the sampling is the most significant factor affecting the airborne microbial community composition. Although seasonal differences have been demonstrated in previous

Printer-friendly version

Discussion paper 
studies, at various locations, I have no knowledge of any that have resulted in such overwhelming differences between two sampling campaigns at the same location, under similar atmospheric conditions. This does not come to doubt the validity of the results; however, extra-caution should be taken to ensure that no confounding variables are responsible for this result. A possible cause for this result might stem from batch effects, e.g., DNA extraction, amplification and sequencing conducted by two different people, on two different occasions might be sufficient in producing such differences. Therefore, the authors are urged to specify whether actions were taken to prevent any batch effects.

Other general suggestions: 1 . The term "seasonality" can be used if a cyclic change over seasons is shown, the difference between May and September of a single year is better referred to as "temporal".

2. Only a single sample per location per month represents the ambient conditions, therefore it is hard to compare dusty to clear conditions. In the absence of several control samples per site, per month, one cannot appreciate the natural variation of the airborne community. With the current study design, the samples representing the same month or the same site cannot provide information on dusty vs. clear days. Possible comparisons can only be made between sites and between sampling periods (September / May). Clear to dusty conditions can only be compared across the entire dataset. However, the great variance observed between May and September probably masks the role of dust in changing the atmospheric bacterial community.

Specific remarks:

L. 32: "concerted succession..." - The use of the term "succession" implies bacterial growth and selection. Please rephrase throughout the manuscript.

Printer-friendly version

L. 54: particle size can well exceed 10 um. Dust storms often carry larger particles (Ryder et al., 2018).

Discussion paper 
L. 61: Please provide a specific website address, the home page of WHO is insufficient.

L. 73-76: Should rephrase: according to the cited paper these genes are not specific to atmospheric bacteria, it is suggested that their presence might enable bacterial survival in the atmosphere.

L. 88: Change "until" to: "up to", or: "reaching".

L. 149-151: this is not so clear. What is the filtering step? What do the two filters represent? A single sampling event? Two consecutive sampling days?

The methods section should clearly indicate how many samples were collected, what was the duration of each sampling event, their dates, etc. It is advised to add a table that sums all the sampling data. DNA extraction and sequencing: The choice of relatively large amplicons ( $\sim 1400 \mathrm{bp}$ ) along with 93 cycles in a paired-end sequencing is surprising. Although there are various platforms that allow pre-processing of nonoverlapping sequences, this might introduce more errors to the alignment step and to the taxonomic classification. Could the very high number of counts per sample (min. 1109571) arise from some sequencing pre-processing error? Were all samples sequenced on a single lane? Were they separated to different lanes according to their dates? Were the amplification and/or sequencing conducted in batches?

\section{182: "OUT table" should be "OTU table"}

Figure 3: Please increase the font and provide clear titles and a color legend within the figure, and not as part of the caption. In general, pie-charts tend to be less clear than bar-charts, since the human eye estimates height differences better than area differences. It is possible to create a dendrogram including all samples, better emphasizing the clustering of samples according to the time of sampling. Also, does each pie-chart represent a single sample or a group of samples? It is not clear how many samples were used for this study.

Printer-friendly version

Section 3.2: The authors describe in detail the differences observed between the sam-

Discussion paper 
ples. These samples represent an array of conditions: dust events vs. clear days; September vs. May; Cagliari vs. Sassari. A clearer representation might be achieved if this section was divided and each comparison was described separately.

L. 315-321: The authors assume that taxa that will show the least variability between two samples of the same dust event, belong to a local core microbiome. This assumption should be better explained, and answer the following questions: 1 . Since dust events introduce new taxa, they are expected to "dilute" the core microbiome, resulting in a variation between dusty/clear conditions. Why not look for the bacteria that decrease in abundance when dust events occur? 2. Comparing two samples of $12 \mathrm{hrs}$ each of a single dust event seems arbitrary. If PM10 mean concentrations throughout the examined $24 \mathrm{hrs}$ remained stable, more dust-related taxa would show low variance, and be considered as "core microbiome". How do the authors assure that this is not the case here?

There is some confusion regarding which taxa were considered as significant for this analysis - L. 316 states that "The latter were considered to represent the common core...", referring to the taxa that showed lower variation. Following, on L. 320, it is stated that: "Only taxa which displayed a mean variation higher than 1/2 of the corresponding standard deviation were considered." In the following tables' captions it is stated that the numbers represent taxa that exceeded the threshold. Please clarify what is the aim of this analysis - to find a core microbiome or to find the "immigrant" bacterial community.

Tables 1 and 2: On table 1 the rows' order represents the two locations, alternately; on Table 2 the rows' order represents first Sassari and then Cagliari. Uniformity between tables is advised.

Table 3: This is the first clear indication of the number of samples taken at each site, under the selected conditions.

Printer-friendly version

Figure 4: When the data is expected to vary along several parameters, it is advised to 
examine other PC axes, e.g., PC3 and PC4. This way, the effect of the most influencing variable (in this case - the time of the sampling) is less expressed, and gives way to see other parameters, possibly. Instead of showing the same PCA triplicated, the authors can attempt to make a more condense view of PC1 vs. PC2, using shapes and colors etc., and add figures displaying other PC axes, if they indeed show some correspondence to the other examined variables.

Figure 5: There's some redundancy in figures and tables: there is no new information arising from the PCoA in Figure 5, that wasn't provided by the PCA in Figure 4. Table 3 provides in detail the diversity indices of the samples, and Figure 5 A and B display it as a boxplot. There's no need for both, the table can be moved to the supplementary information.

L. 416: Using a Bonferroni correction for multiple comparisons is unnecessarily stringent. It resulted in only 6 taxa that were significantly differentially abundant when comparing the two sampling periods. A result of only six significant genera is probably of a low ecological relevance. It is more advised to apply Benjamini-Hochberg correction to achieve better statistical power, and a greater collection of significant taxa. It is often the case with very low $p$-values, that they represent rare taxa that are found in very few samples. I advise the authors to inspect the six significant taxa, and make sure this is not the case here. Moreover, other statistical tools, better fitting microbiome data analysis, are available for differential abundance tests, e.g., ANCOM, ANCOM-BC, MaAsLin2, etc. These methods should be preferred over ANOVA tests for compositional datasets.

Table 5: This table is somewhat overcrowded and demands an intense reading to draw conclusions from. It presents pair-wise distances between individual samples, and not between groups of samples (May vs. September, CA vs. SS, etc.), which provides no statistical significance to the observations arising from it. It also seems somewhat redundant, there is no added value in this table over the dendrogram, PCA and PCoA already presented.

Printer-friendly version

Discussion paper 
L. 473: the declared goal of the study is indeed significant to the understanding the processes affecting the airborne microbial community in the Mediterranean; however, the design of the study suffers from too few samples. There are too few controls, and only two sampling campaigns, representing two seasons. Seasonality cannot be determined without repetitive sampling during the same season over several years. The presented differences can be referred to as temporal.

L. 484: This remark is very true.

L. 488: This finding is very different from what was suggested by others before, e.g., Gat et al. (2017); Lang-Yona et al. (2020); Bowers et al. (2013); Caliz et al. (2018). Seasonal variations were shown in previous studies, yet they were not as extreme as shown in this study. Sampling location and aerosol back-trajectories were usually more significant in determining the airborne community composition. Did the authors make sure that no batch effects or other confounding variables stand behind the results presented here?

L. 527-532: The referral to PM10 concentrations is significant, especially when considering the changes in community composition during dust events. According to Figure S1, the dust events on May and September have tripled the atmospheric PM10 concentrations, compared with clear days. This significant change in PM10 is expected to result in a significant change in the airborne bacterial community (see Mazar et al., 2016). Yet, this change seems minor according to Fig. 3, 4 and 5. How do the authors explain this result?

L. 551-554: As stated before, sometimes the lowest $p$-values are given by the rarest taxa. Please make sure this is not the case for these taxa.

L. 576-593: Due to the low number of control samples, it is impossible to draw conclusions on dusty vs. clean conditions of the same season or the same location.

Printer-friendly version

Discussion paper

Interactive comment on Biogeosciences Discuss., https://doi.org/10.5194/bg-2020-324, 2020. 